\title{
Morus alba and active compound oxyresveratrol exert anti-inflammatory activity via inhibition of leukocyte migration involving MEK/ERK signaling
}

Yi-Ching Chen ${ }^{1 \dagger}$, Yin-Jing Tien ${ }^{2+}$, Chun-Houh Chen ${ }^{2}$, Francesca N Beltran ${ }^{3}$, Evangeline C Amor ${ }^{3}$, Ran-Juh Wang ${ }^{4}$, Den-Jen $\mathrm{Wu}^{4}$, Clément Mettling ${ }^{5}$, Yea-Lih Lin ${ }^{5}$ and Wen-Chin Yang ${ }^{1,6,7,8^{*}}$

\begin{abstract}
Background: Morus alba has long been used in traditional Chinese medicine to treat inflammatory diseases; however, the scientific basis for such usage and the mechanism of action are not well understood. This study investigated the action of M. alba on leukocyte migration, one key step in inflammation.

Methods: Gas chromatography-mass spectrometry (GC-MS) and cluster analyses of supercritical $\mathrm{CO}_{2}$ extracts of three Morus species were performed for chemotaxonomy-aided plant authentication. Phytochemistry and CXCR4-mediated chemotaxis assays were used to characterize the chemical and biological properties of M. alba and its active compound, oxyresveratrol. fluorescence-activated cell sorting (FACS) and Western blot analyses were conducted to determine the mode of action of oxyresveratrol.

Results: Chemotaxonomy was used to help authenticate M. alba. Chemotaxis-based isolation identified oxyresveratrol as an active component in M. alba. Phytochemical and chemotaxis assays showed that the crude extract, ethyl acetate fraction and oxyresveratrol from M. alba suppressed cell migration of Jurkat T cells in response to SDF-1. Mechanistic study indicated that oxyresveratrol diminished CXCR4-mediated T-cell migration via inhibition of the MEK/ERK signaling cascade.

Conclusions: A combination of GC-MS and cluster analysis techniques are applicable for authentication of the Morus species. Anti-inflammatory benefits of $M$. alba and its active compound, oxyresveratrol, may involve the inhibition of CXCR-4-mediated chemotaxis and MEK/ERK pathway in T and other immune cells.
\end{abstract}

Keywords: Chemotaxis, CXCR4, Morus, Phytochemistry and T-cells

\section{Background}

The genus Morus from the Moraceae family consists of 10-16 species of deciduous trees that are distributed worldwide [1]. Different parts of the Morus plants such as leaves, fruit, branches, bark, root, and shoot have been used as food and herbal medicine in China for over 1900 years [2]. In Taiwan, M. alba, commonly known as white mulberry, is possibly the Morus species most frequently used in traditional Chinese medicine

\footnotetext{
* Correspondence: wcyang@gate.sinica.edu.tw

${ }^{\dagger}$ Equal contributors

${ }^{1}$ Agricultural Biotechnology Research Center, Academia Sinica, 128, Sec. 2,

Academia Road, Nankang, Taipei 11501, Taiwan

${ }^{6}$ Department of Life Sciences, National Chung Hsing University, Taichung 402, Taiwan

Full list of author information is available at the end of the article
}

although it is sometimes used interchangeably with $M$. atropurpurea and M. bombycis. As all three species have similar morphological characteristics and habitat preferences they are frequently misidentified. $M$. alba, the most extensively studied species, has been reported to have anti-hyperlipidemic [3], anti-hypertensive [4,5], anti-hyperglycemic [2,6]; Hansawasdi, 2006; [7], antimicrobial [8-12], anti-allergic [13], anti-inflammatory $[14,15]$, hepatoprotective $[16,17]$, neuroprotective [18], immuno-modulatory [19], and anti-venom activities [20].

Photochemical studies have identified alkaloids, flavonoids, flavones, flavanones, stilbenes, benzophenones, coumarin derivatives and terpenoids in Morus species [5,21-37]. These compounds are likely responsible for the bioactivities of the 
Morus plants. Among them, the stilbenes, oxyresveratrol and resveratrol, were reported present in the Morus plants and demonstrated antioxidant activity [38]. Oxyresveratrol inhibited nitrogen oxide (NO) production, inducible $\mathrm{NO}$ synthase (iNOS) expression, prostaglandin E2 ( $\left.\mathrm{PGE}_{2}\right)$ production, and activation of nuclear factor kappa-light-chain enhancer of activated B cells (NFkB) in macrophages [39]; and consistently reduced edema induced by carrageenan in a mouse model [39]. The above data suggest the involvement of oyxresveratrol in suppression of the inflammatory process. Nevertheless, the role of oxyresveratrol in the regulation of leukocyte migration has not been studied.

Migration of leucocytes from blood vessels to the flamed sites is a fundamental feature of inflammation. Chemokines and chemokine receptors can orchestrate leukocyte migration, also termed chemotaxis [40]. In addition to its physiological functions, chemotaxis is implicated in inflammation and disease pathogenesis [41]. Therefore, chemotaxis has been proposed as a key target of anti-inflammatory drugs [42]. CXCR4, a G-protein-linked transmembrane receptor, is expressed in all leukocytes, blastocysts and a variety of cancer cells [43]. SDF-1 (CXCL12) is a natural ligand of CXCR4. After binding to SDF-1, CXCR4 triggers a signaling cascade which includes the activation of kinases (FAK, PI3K, ERK, JAK, and TYK) and downstream molecules (NFKB and STAT) and, eventually regulates chemotaxis, locomotion, and adhesion [43].

Plants provide an extraordinary source of lead compounds for a myriad of disorders including inflammation. In this study, we investigated the role of $M$. alba in leukocyte migration, a key step in inflammation. As M. alba is easily misidentified due to its close resemblance to other Morus plants, we first devised a chemotaxonomic strategy involving GC-MS analysis and cluster analysis of the chemical profiles to differentiate between the Morus species. Next, the anti-chemotactic activities of $M$. alba and its active compound, oxyresveratrol, were examined in Jurkat T cells. Finally, the mode of action of $M$. alba and its active compound, oxyresveratrol, was investigated.

\section{Methods}

\section{Reagents}

High performance liquid chromatography (HPLC) grade solvents, acetonitrile (ACN), dichloromethane, butanol, methanol and ethyl acetate were purchased from Avantor Performance Materials (NJ, USA). Trifluoroacetic acid (TFA), methanol, dimethyl sulfoxide (DMSO), resveratrol and oxyresveratrol were purchased from Sigmal (MO, USA). RPMI 1640 medium, PSQ solution (penicillin, streptomycin and glutamine), sodium pyruvate, nonessential amino acids and HEPES were purchased from Gibco (CA, USA). $\alpha$ CXCR4, FITC-conjugated secondary antibody (Life Technologies, NY, USA) and SDF-1 (R\&D systems, MN, USA) were purchased. WST-1 reagent was purchased from Roche (Mannheim, Germany). Antibodies against mitogen-activated protein kinases (MAPKs) and their phosphorylated proteins were purchased from Cell Signaling Technology (MA, USA).

\section{Supercritical liquid extraction and GC-MS analysis of plants} M. alba, $M$. atropurpurea and $M$. bombycis were collected and authenticated by Dr Ran-Juh Wang from the Miaoli District Agricultural Research and Extension Station (MDARES), Miaoli County, Taiwan, in 2010. Their voucher specimens were deposited as No. 00083241, No. 00082146, and No. 00083303, respectively, at the MDARES herbarium. Branches of the plants were air dried and pulverized. Five grams of plant samples were extracted with carbon dioxide using the supercritical fluid extractor SFX System 1220R (ISCO, NE, USA), followed by GC-MS analysis using a Trace gas chromatograph interfaced to a Polaris Q mass spectrometer (Thermo Finnigan, Hertfordshire, UK) in EI mode (70 eV) as previously described [44].

\section{Extract preparation, HPLC and compound identification}

Branches of Morus plants were pulverized and extracted with methanol $(2 \times 3 \mathrm{~L})$. The methanol extracts were evaporated by rotary evaporator, yielding $1 \mathrm{~g}$ of crude extracts. The $M$. alba crude extract was re-suspended with water $(150 \mathrm{~mL})$, followed by sequential extraction with ethyl acetate $(9 \times 150 \mathrm{~mL})$ and butanol $(6 \times 150 \mathrm{~mL})$. Crude extracts, fractions, and oxyresveratrol were analyzed using an Agilent 1100 Series HPLC system. HPLC was performed on a Luna C18 column (Phenomenex, CA, USA) at a flow rate of $0.5 \mathrm{ml} / \mathrm{min}$ and $25^{\circ} \mathrm{C}$ with a photodiode detector at $254 \mathrm{~nm}$. The solvent gradient for HPLC was set in the following sequence, $10 \%$ to $50 \%$ of $\mathrm{B}$ for 0-50 $\mathrm{min}, 50 \%$ of B for $50-65 \mathrm{~min}, 50$ to $100 \%$ of B for 65-70 $\mathrm{min}$, and $100 \%$ of $\mathrm{B}$ for 70 to $95 \mathrm{~min}$. Solvents $\mathrm{A}$ and $\mathrm{B}$ were $0.05 \% \mathrm{TFA} / \mathrm{H}_{2} \mathrm{O}$ and $0.05 \% \mathrm{TFA} / \mathrm{CAN}$, respectively. The identity of oxyresveratrol with a purity of $97 \%$ from $M$. alba was confirmed by comparing its spectroscopic data with previously published data $[45,46]$.

\section{Chemotaxis assay and WST-1 assay}

Jurkat cells E6.1 (ATCC No. TIB-152), a leukemic T cell line, were cultured in complete medium as described [47]. The cells $\left(1 \times 10^{6} / \mathrm{mL}\right)$ were pre-treated with crude extract, fractions, or compounds of $M$. alba for $24 \mathrm{~h}$ in $1 \%$ FBS medium. The cells were transferred into a transwell insert (Millipore, USA) and put into a 24-well plate where SDF-1 or vehicle (PBS) was added to the $1 \%$ FBS medium. After $4 \mathrm{~h}$, the cells that migrated to the bottom of the 24-well plate were counted and photographed. The migration index (MI) was defined 


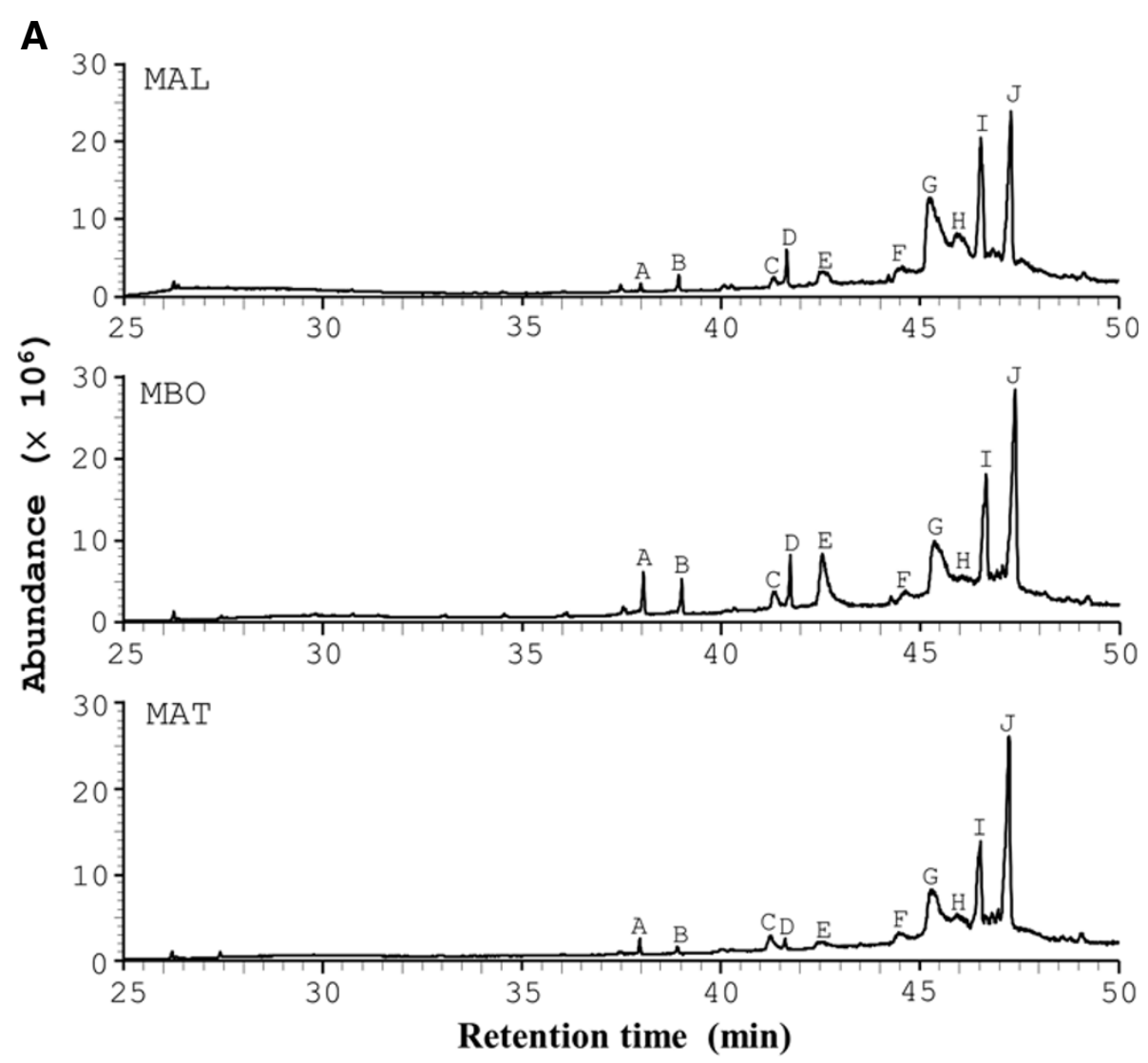

B

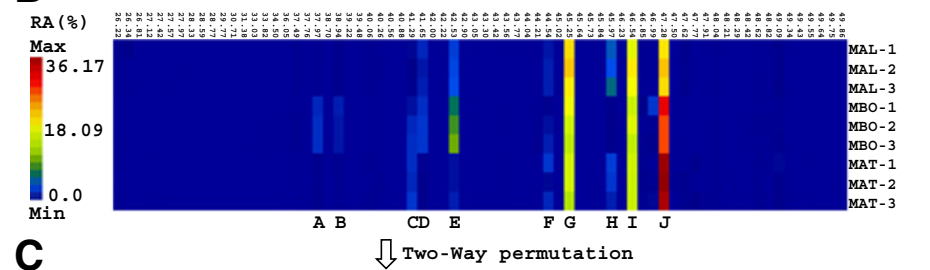

C

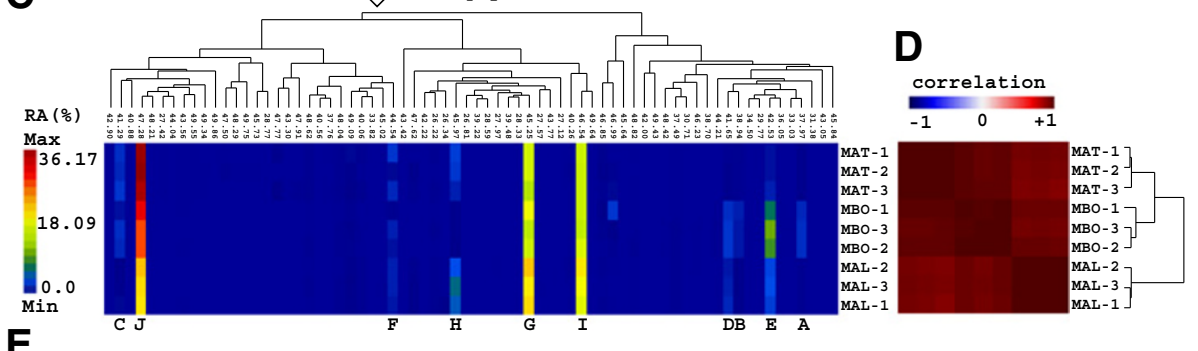

E

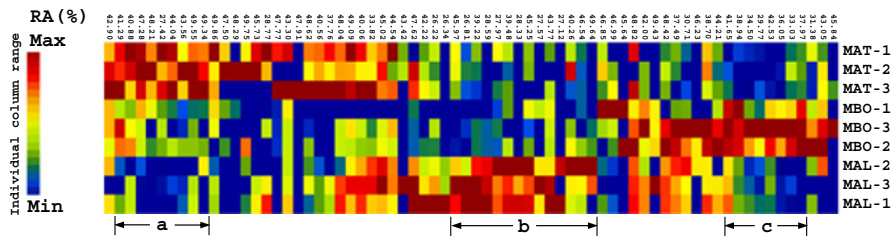

Figure 1 (See legend on next page.) 
(See figure on previous page.)

Figure $1 \mathrm{GC}-\mathrm{MS}$ profiles and cluster analysis of the three Morus species. (A) Branches of M. alba (MAL), M. atropurpurea (MAT), and M. bombycis (MBO) underwent supercritical $\mathrm{CO}_{2}$ extraction and GC-MS analysis at a dose of $10 \mathrm{mg} / \mathrm{ml}$. One representative GC-MS chromatogram from 3 batches of each plant extract is shown. The sizes of the various GC-MS peaks are measured as percent relative area (RA (\%)) of the largest GC-MS peak in the chromatogram. (B) Matrix visualization with hierarchical cluster trees (HCT) for GC-MS profiles of the Morus species.

A representative GC-MS profile of the 3 Morus species from Figure 1 is displayed as a matrix map with 9 rows, each representing one sample, and 70 columns, each representing one retention time. A rainbow spectrum was used to color code the size of the chromatographic peaks (expressed in percent relative area, RA (\%)) in the whole matrix. Red denotes the largest peak (maximum RA (\%)) and blue denotes the smallest peak (minimum RA (\%)). (C) Two-way permuted data matrix of (B). The tree structure above the data matrix represents average linkage hierarchical cluster tree (HCT) for 70 different retention times. (D) Map of correlation matrix for the 9 samples (three samples per species). A bi-directional blue-white-red spectrum is used to denote negative-to-positive correlation. The tree structure besides the correlation matrix represents average linkage HCT for 9 samples. (E) Identical data matrix map as in (B) except each column is colored using a rainbow color spectrum scaled individually for that particular column instead of a rainbow color spectrum scaled for all columns in the matrix.

below. MI $(\%)=100 \times($ number of drug-treated cells migrating toward SDF-1 minus number of drug-treated cells migrating toward PBS)/(number of vehicle-treated cells migrating toward SDF-1 minus number of vehicletreated cells migrating toward PBS) [47]. For WST-1 assay, Jurkat cells $\left(1 \times 10^{6} / \mathrm{mL}\right)$ were incubated with the crude extract, fractions and compound for $24 \mathrm{~h}$. After PBS washing, the cells were incubated with WST-1 for $1 \mathrm{~h}$ and measured at $440 \mathrm{~nm}$ using the BioTek ELISA reader (VT, USA).

\section{Western blot and FACS analysis}

Jurkat cells were pre-treated with vehicle and compounds at the indicated concentrations for $1 \mathrm{~h}$. For Western blot, the cells were stimulated with SDF-1 for the indicated time. Total lysates underwent sodium dodecyl sulfate polyacrylamide gel electrophoresis and blotting with the indicated antibodies, followed by ECL visualization. To determine the CXCR4 expression level, the cells were stained with $\alpha$ CXCR4 and secondary antibody and underwent FACS analysis using FlowJo software.

\section{Statistical analysis}

Data from three experiments or more are presented as mean \pm standard deviation. Comparisons between experimental groups and control were made using ANOVA. $P$ values (*) less than 0.05 were considered statistically significant.

\section{Results}

\section{GC-MS chromatograms and cluster analysis of the chemical profiles of Morus species}

Correct authentication of medicinal plants is an essential prerequisite to ensure reproducible quality and efficacy of herbal medicine. M. alba, M. atropurpurea and M. bombycis are all common in Taiwan. However, their dried samples and products are frequently indistinguishable. For this purpose, a combination of GC-MS and cluster analysis tools were used to rapidly distinguish the three Morus species. First, GC-MS analysis of the supercritical $\mathrm{CO}_{2}$ extracts was used to profile the chemical compositions of the three species (Figure 1A). Peaks A to $\mathrm{J}$ are shown in Figure 1. As previously described $[48,49]$, cluster analysis tools, such as hierarchical cluster trees (HCT), principal component analysis (PCA) and multidimensional scaling (MDS), were next used to analyze the inter- and intra-species chemical variations. In this work, we adopted HCT with matrix visualization (MV) in generalized association plots (GAP) [50,51] to compare the similarities and differences in the chemical profiles of the Morus extracts (Figure 1A). Figure 1B shows the matrix visualization of the sizes of the GCMS peaks in percent relative areas [RA (\%); area of a GC-MS peak normalized against the area of the largest peak in the chromatogram, presented as a percentage] of 9 samples (3 samples of each species) with 70 retention times between $26.22 \mathrm{~min}$ and $49.86 \mathrm{~min}$. A rainbow spectrum was used to color code the sizes of the chromatographic peaks with red denoting the largest peak [maximum RA(\%)] and blue denoting the smallest peak [minimum RA(\%)]. Each row in Figure 1B represents one sample while each column represents one particular retention time. Average linkage hierarchical cluster trees (HTCs) for retention time and for samples were then built on corresponding correlation matrices for retention time and samples, respectively. Figure $1 \mathrm{C}$ is obtained from the two-way permutation according to the orders of terminal nodes in HCTs for retention time and samples in order to obtain sample cluster structure and retention time grouping pattern [51]. In Figure 1D, a bi-directional blue-white-red spectrum is used to denote negative-to-positive correlation. The most profound observation to be noted in Figure 1D was that $M$. atropurpurea and M. bombycis samples form a larger group with high positive within-group correlation (in dark red) while $M$. alba samples form a separate cluster.

By converting Figure $1 \mathrm{C}$ to Figure $1 \mathrm{E}$ using individually ranged color spectra for each retention time so that between-variant structure within each retention time (column) can be clearly depicted, we found that the combination of matrix visualization for GC-MS chemical profiles with hierarchical cluster trees using GAP was a 
fast and reliable chemotaxonomical method to assist in the identification of the three Morus species. In Figure 1E, the GC-MS peaks of $M$. alba (MAL), M. atropurpurea (MAT) and $M$. bombycis (MBO) are relatively larger than others with retention time in groups $\mathrm{a}, \mathrm{b}$ and $\mathrm{c}$, respectively. By selecting the components of groups $\mathrm{a}, \mathrm{b}$, and $\mathrm{c}$ in Figure $1 \mathrm{E}$ and significant relative peak areas in Figure $1 \mathrm{C}$, we identified peaks J, G, I, and E (Figure 1C) as useful parameters to distinguish between the three species.

Overall, the combination of GC-MS and cluster tools is feasible and suitable for determining the taxonomy of the Morus species.

\section{HPLC profiles of the three Morus plant extracts}

Having authenticated the M. alba, M. atropurpurea, and $M$. bombycis branches, we next sought to characterize their chemical properties. HPLC is a useful method for analysis and comparison of chemical fingerprints of plant extracts. HPLC analysis showed similar and distinct peaks among the 3 Morus species (Figure 2). The peaks at the retention times, 14.0, 31.3, 64.7, 74.0 and $77.6 \mathrm{~min}$, appeared in all three species despite the difference in the relative amount of the peaks. Of note, peak 1 at the retention time of 31.3 min was present in the crude extracts of the three Morus species. In contrast, M. alba had a more abundant peak 1 than $M$. atropurpurea and $M$. bombycis. Peak 1 was isolated from the $M$. alba extract and identified as oxyresveratrol as shown in Figure 3. The NMR data (Additional file 1: Table S1), UV spectra data
(Additional file 1: Figure S1A) and MS data (Additional file 1: Figure S1B) of oxyresveratrol in the study were analyzed and confirmed with those described elsewhere [45,46].

\section{Characterization of $M$. alba extract using a combination of phytochemical and chemotaxis assays}

$M$. alba has long been used as anti-inflammatory medicine. To further understand the anti-inflammtory activity of $M$. alba, a chemotaxis-guided fractionation and isolation strategy was adopted (Figure 3A). HPLC analysis of the crude extract, fractions and oxyresveratrol, an active compound, of $M$. alba showed that oxyresveratrol (peak 1) was present in the crude extract (CE, Figure $3 \mathrm{~B}$ ) and ethyl acetate fraction (EA, Figure 3B) of $M$. alba; however, oxyresveratrol was not detectable in the butanol $(\mathrm{BuOH}$, Figure 3B) and water $\left(\mathrm{H}_{2} \mathrm{O}\right.$, Figure 3B) fractions of $M$. alba.

Examination of the effect of the crude extract, fractions and oxyresveratrol of $M$. alba on CXCR4mediated chemotaxis further showed that the $M$. alba crude extract and the ethyl acetate fraction inhibited chemotaxis in Jurkat $\mathrm{T}$ cells, key players in immune response (Figure 4A). In contrast, the butanol and water fractions of $M$. alba did not inhibit CXCR4-mediated chemotaxis (Figure 4A). Consistently, oxyresveratrol dose-dependently inhibited CXCR4-mediated chemotaxis in T cells (Figure 4B). This inhibition by the crude extract, fractions and oxyresveratrol of $M$. alba was not due to cytotoxicity because treated cells showed high viability (Figure 4C). Overall, the data suggest that
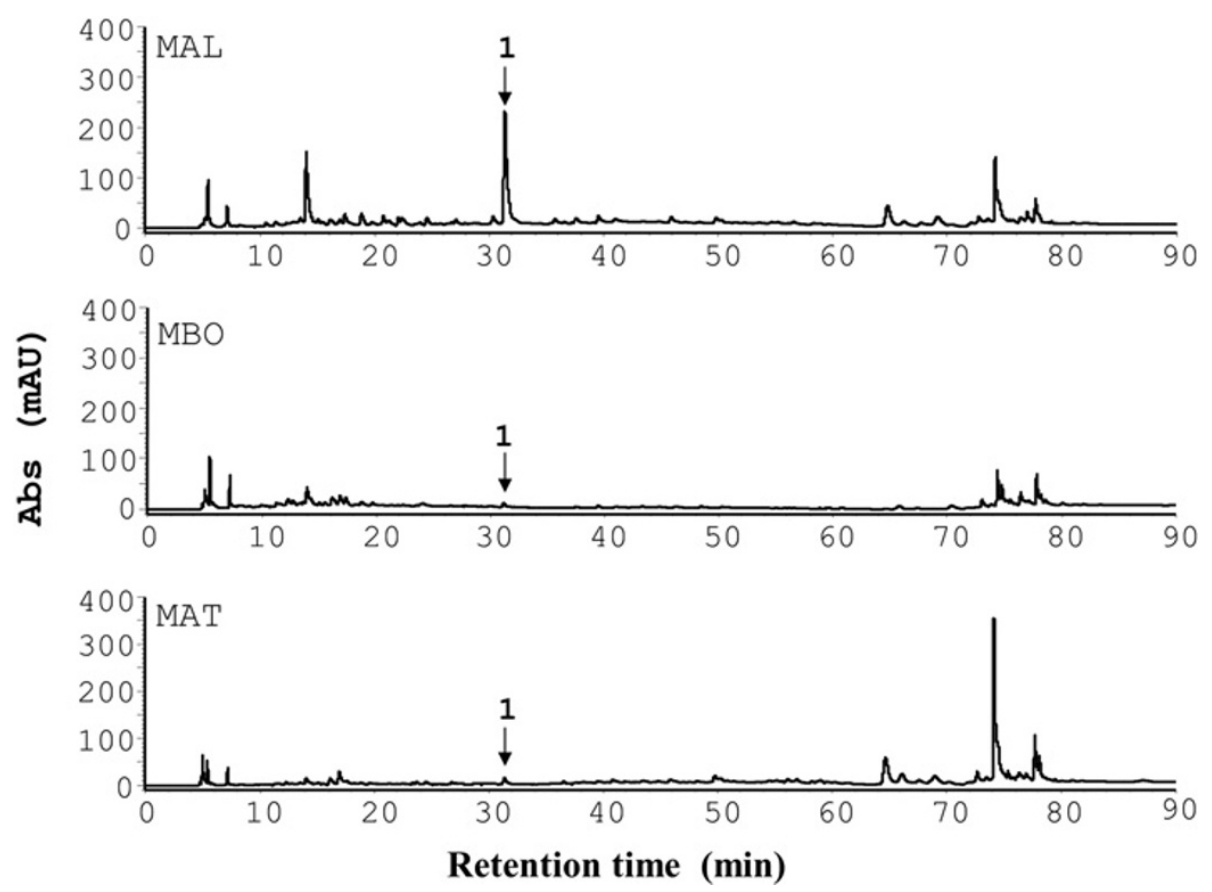

Figure 2 HPLC profiles of the crude extracts of the three Morus species. Methanol crude extracts of each species at a dose of $25 \mathrm{mg} / \mathrm{ml}$ were analyzed using an RP-18 HPLC column and detected with a UV detector at $254 \mathrm{~nm}$. Peak 1 (oxyresveratrol) is indicated. 


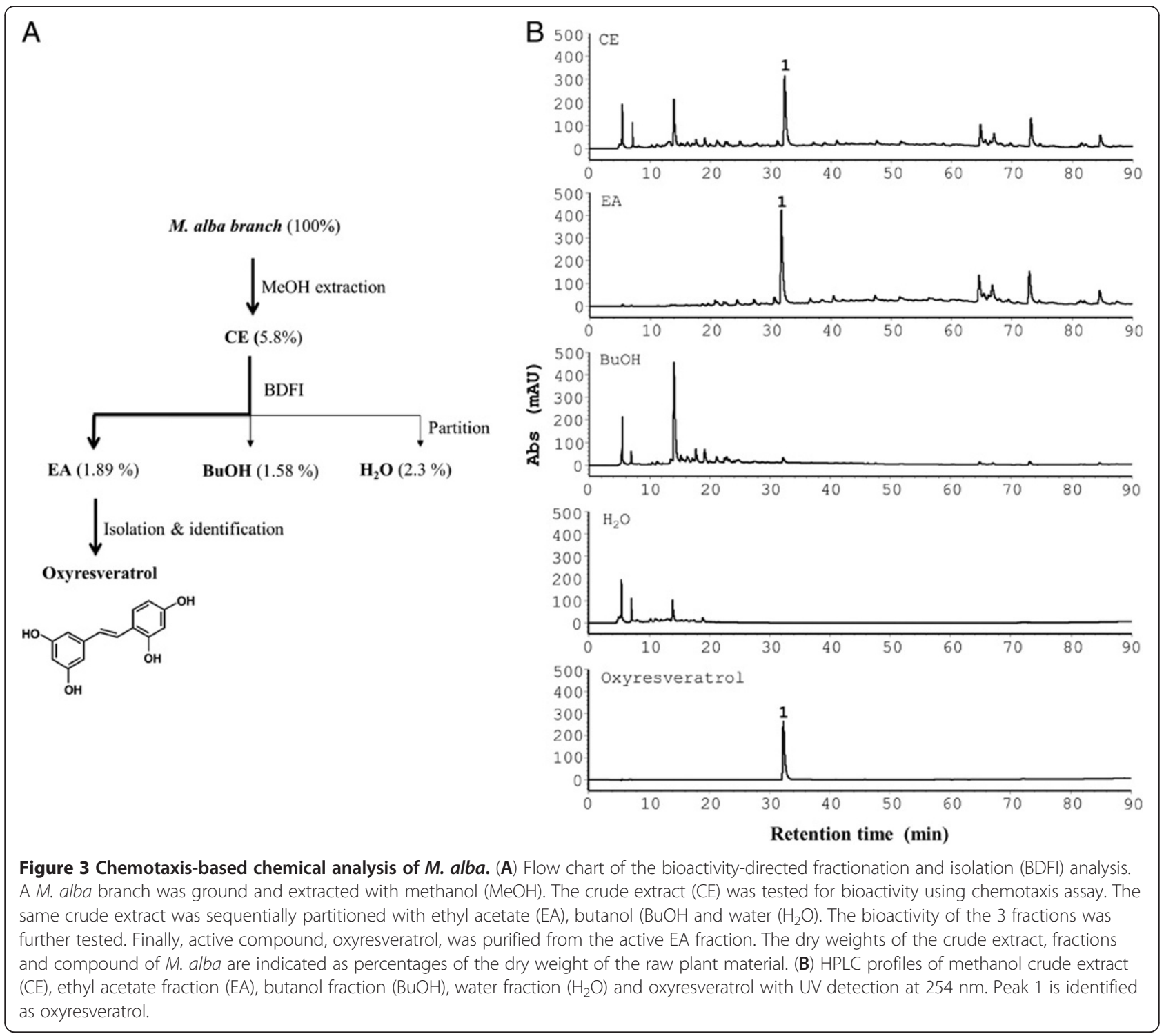

M. alba and oxyresveratrol suppress inflammation via inhibition of leukocyte migration.

\section{Mechanistic study of oxyresveratrol in CXCR4-mediated chemotaxis}

To dissect the mechanism by which oxyresveratrol inhibited leukocyte migration, we first examined whether oxyresveratrol could down-regulate the level of cell surface expression of the chemokine receptor CXCR4. Jurkat $\mathrm{T}$ cells were treated with oxyresveratrol and the CXCR4 expression level on $\mathrm{T}$ cells was measured using FACS analysis. FACS data showed no difference in the surface expression of CXCR4 on cells treated with oxyresveratrol, resveratrol, or DMSO vehicle (Figure 5A), suggesting that oxyresveratrol does not act at the chemokine receptor level.
Next, we investigated the impact of oxyresveratrol on the CXCR4 downstream signaling molecules. MAPKs are known to function downstream of chemokine receptors [47]; therefore, we examined whether oxyresveratrol influenced MAPKs in the CXCR4 pathway. Western blot data showed that oxyresveratrol inhibited SDF-1-mediated phosphorylation of ERK1/2 kinases (Figure 5B). Conversely, it enhanced the SDF-1-mediated phosphorylation of p38 (Figure 5B). However, oxyresveratrol did not appear to affect SDF-1-mediated JNK phosphorylation (Figure 5B). Knowing that oxyresveratrol inhibited the activation of ERK1/2, we further examined the effect of oxyresveratrol on the upstream activators of ERK1/2 kinases, MEK1/2 kinases. Accordingly, it suppressed the phosphorylation of MEK1/2 kinases in the CXCR4 pathway (Figure 5C). These data demonstrated that oxyresveratrol in $M$. alba suppresses CXCR4-mediated chemotaxis via inactivation of 
A

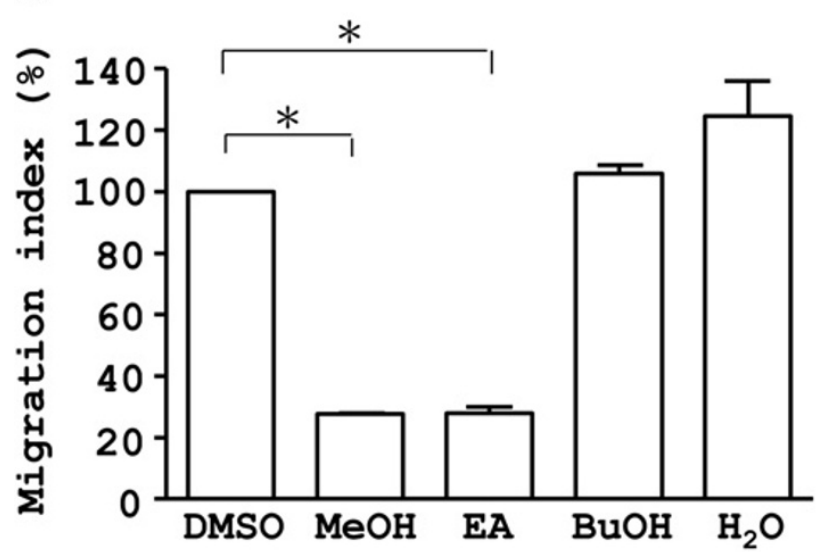

B

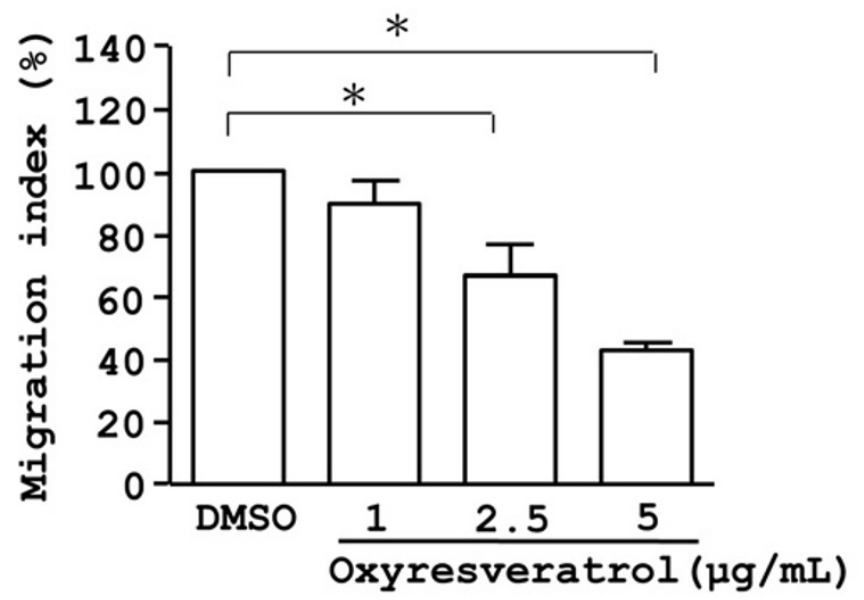

C

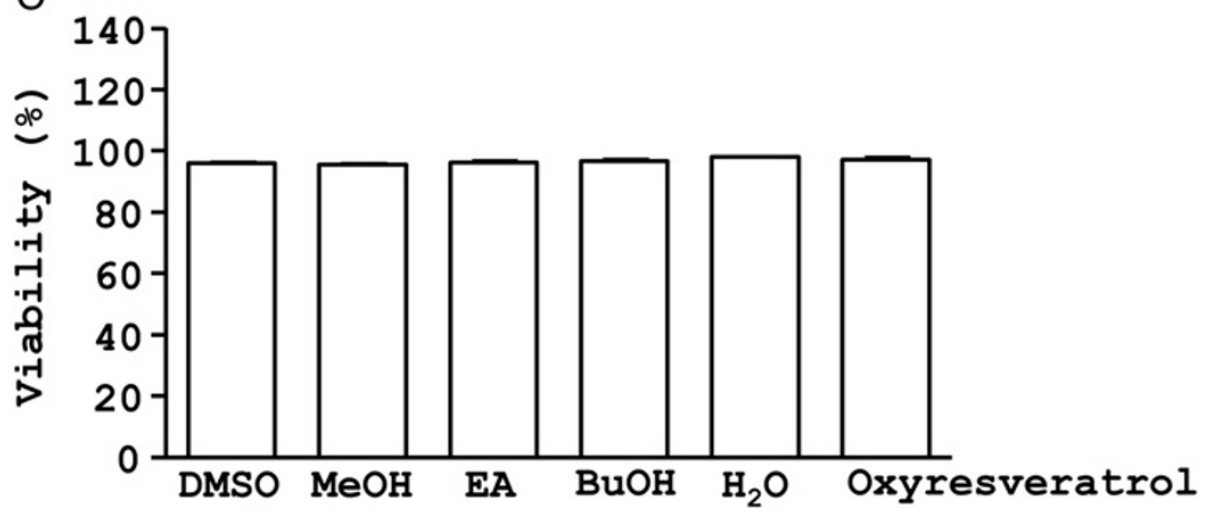

Figure 4 Effect of crude extract, fractions and compound of $\boldsymbol{M}$. alba on chemotaxis and cell viability. (A) Jurkat cells were pre-treated with vehicle (DMSO), the crude extract $(C E, 10 \mu \mathrm{g} / \mathrm{ml})$, butanol fraction $(\mathrm{BuOH}, 4 \mu \mathrm{g} / \mathrm{ml})$, ethyl acetate fraction (EA, $4 \mu \mathrm{g} / \mathrm{ml})$ and water fraction $\left(\mathrm{H}_{2} \mathrm{O}, 10 \mu \mathrm{g} / \mathrm{ml}\right)$ of $\mathrm{M}$. alba for $24 \mathrm{~h}$. The cells were treated with PBS and SDF-1, respectively, for an additional $4 \mathrm{~h}$ in transwell microplates. The number of cells in the bottom well was counted. Cell migration is indicated as migration index (\%), as defined in Materials and methods. (B \& C) Jurkat cells were pre-treated with oxyresveratrol at 1,2.5 and $5 \mu \mathrm{g} / \mathrm{ml}$. The cells underwent chemotaxis assays (B) or WST-1 test (C) for cell viability. $P\left({ }^{*}\right)<0.05$ is considered statistically significant. 


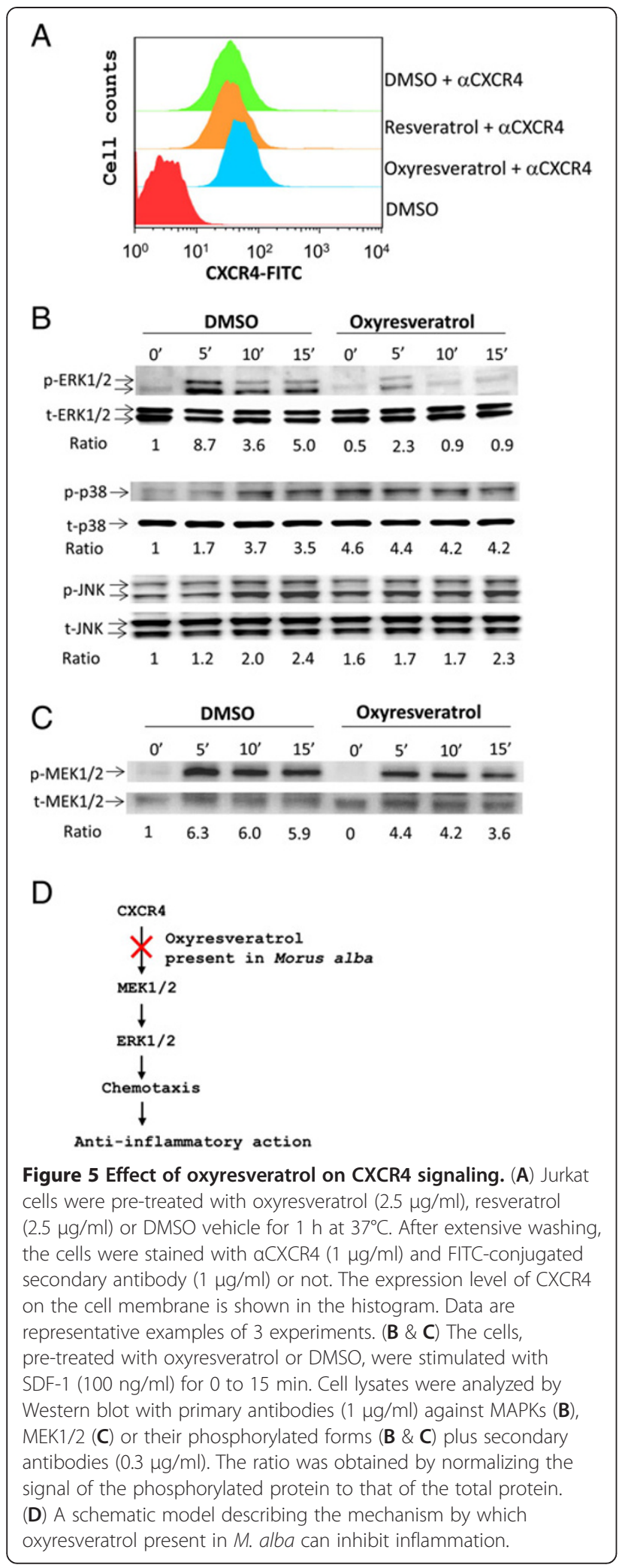

the ERK signaling pathway. The data on Jurkat cells can be confirmed with normal leukocytes. Moreover, overall data suggest that anti-inflammatory herb, M. alba, and its oxyresveratrol suppress inflammation via inhibition of leukocyte migration involving the MEK/ERK pathway (Figure 5D).

\section{Discussion and conclusions}

Correct identification and authentication of plants are absolutely necessary for batch consistency and therapeutic efficacy of botanical medicines. $M$. alba is used in traditional Chinese medicine for treatment of inflammatory disorders. However, M. alba is sometimes used interchangeably with, or confused with other Morus species such as $M$. atropurpurea and $M$. bombycis. A standard method of identification of $M$. alba versus $M$. atropurpurea or $M$. bombycis has not been established, and the similarities/differences between the chemical and biological properties of these three species have not been studied. In this work, we first used chemotaxonomic methods to differentiate $M$. alba from the other two species. Spectroscopic methods were used to analyze the chemical fingerprint of crude extracts, fractions, and compounds of M. alba. Combined photochemistry and chemotaxis assays were then used for identification and anti-inflammatory study of $M$. alba and oxyresveratrol.

Chemokines and their receptors are involved in numerous diseases and have roles in inflammation and infection. Consequently, the antagonists and inhibitors of the chemokines and their receptors have become potential drug targets for inflammatory diseases [41]. M. alba has been long used as anti-inflammatory remedy in China. Nevertheless, little is known about its role in leukocyte migration, a central process linked to inflammation, infection and disease pathogenesis. Here, we demonstrated that $M$. alba can suppress leukocyte migration triggered by CXCR4 (Figure 4). Unexpectedly, bioassay-guided isolation and identification yielded oxyresveratrol as the major active component of the bioactive ethyl acetate fraction of M. alba (Figure 3). $M$. alba possessed a higher abundance of oxyresveratrol than the other Morus plants (Figure 2). The evidence presented here that $M$. alba and its active component, oxyresveratrol, suppress CXCR4-mediated leukocyte migration supports the traditional use of $M$. alba as an anti-inflammatory medicine.

Oxyresveratrol has been previously reported to exert anti-inflammatory activity through inhibition of iNOS/NO production, PGE2 synthesis and NFKB activation [39]; and was reported to reduce carageenan-induced paw edema in rats likely through inhibition of iNOS expression [39]. We have also observed oxyresveratrol to be a more potent inhibitor of leukocyte migration than 
resveratrol (Figure 4B and data not shown). Our data on the difference in chemotactic action between resveratrol and oxyresveratrol are in good agreement with a previous publication indicating that resveratrol does not inhibit chemotaxis [47]. Thus, the $\mathrm{OH}$ functional group in oxyresveratrol appears to be responsible for its anti-chemotactic activity. Furthermore, oxyresveratrol inhibited the activation of the MEK/ERK pathway (Figure 5), suggesting a possible mechanism by which $M$. alba and oxyresveratrol inhibit inflammation.

Following engagement, chemokine receptors induce an activation of $\mathrm{G}$ proteins, tyrosine kinases, serine/ threonine kinases and phospholipases, leading to cell migration $[52,53]$. Our results showed that oxyresveratrol, isolated from M. alba, inhibited the activation of MEK/ ERK kinases, a serine/threonine kinase family, mediated by CXCR4 in T-cells (Figure 5). However, oxyresveratrol did not affecte the expression level of CXCR4 receptor (Figure 4A). These findings suggest that unlike the receptor antagonists, oxyresveratrol targets the intraceullar proteins downstream of the chemokine receptors and may be used as alternative inhibitors of chemokine signaling. Consistently, MAPKs are known to modulate inflammatory responses and are thought to be attractive molecular targets for anti-inflammatory therapy [54]. The manifestation of inactivation of MEK/ERK pathway by oxyresveratrol makes it extremely interesting potential natural anti-inflammatory remedy.

In conclusion, we combined phytochemical and chemotaxis techniques to study the anti-chemotactic role of the Morus plants. We demonstrated that M. alba and its active compound, oxyresveratrol, suppress inflammation via inhibition of leukocyte chemotaxis. Mechanistic studies showed that oxyresveratrol inhibits CXCR4-mediated leukocyte migration via inactivation of the MEK/ERK pathway downstream of the CXCR4 receptor. These findings support the claims of the benefits of $M$. alba purported in traditional Chinese medicine and suggest the possible use of the active compound oxyresveratrol as an anti-inflammation therapy.

\section{Additional file}

Additional file 1: Table S1. ${ }^{1} \mathrm{H}$ and ${ }^{13} \mathrm{C}$ NMR data on oxyresveratrol dissolved in $\mathrm{CD}_{3} \mathrm{COCD}_{3}$. Figure S1. Ultraviolet (UV) spectra and mass spectrometry data of oxyresveratrol present in the crude extract and ethyl acetate fraction of Morus alba and oxyresveratrol. (A) The crude extract (CE) and ethyl acetate (EA) fraction and resveratrol were subjected to high performance liquid chromatography (HPLC) and detected with a diode array detector at $254 \mathrm{~nm}$ as described in the Materials and methods section. The UV spectra of resveratrol (peak 1) are indicated. Peak 1 corresponds to the same peak as Figure B. (B) Electrospray ionization mass spectrometry (ESI-MS) spectra of oxyresveratrol present in the crude extract and ethyl acetate fraction of M. alba and oxyresveratrol. The crude extract and ethyl acetate fraction of $M$. alba and oxyresveratrol were subjected to HPLC-ESI-MS. The MS scans were performed in negative ion mode (m/z 200 to $\mathrm{m} / \mathrm{z}$ 400). Peaks 1 (31.3 min) of the crude extract (CE) and ethyl acetate fraction (EA) of $M$. alba and oxyresveratrol showed ion signals at $\mathrm{m} / \mathrm{z} 243$. Peak 1 corresponds to the same peak as Figure 3B.

\section{Abbreviations}

FACS: Fluorescence-activated cell sorting; GC-MS: Gas chromatography-mass spectrometry; HPLC: High performance liquid chromatography; iNOS: Inducible NO synthase; NFkB: Nuclear factor kappa-light-chain enhancer of activated B cells; NO: Nitrogen oxide; $\mathrm{PGE}_{2}$ : Prostaglandin E2.

\section{Competing interest}

The authors declare that they have no competing interests.

\section{Authors' contributions}

YLL, CM and WCY conceived the study. YCC, YJT and FNB carried out the experiments. $\mathrm{CHC}$ and ECA helped YCC, YJT and FNB in carrying out the experiments. RJW and DJW participated in designing the experiments and plant authentication. WCY wrote the manuscript. All authors read and approved the final manuscript.

\section{Acknowledgements}

This work was supported by the National Core Facility Program for Biotechnology, Taiwan (Bioinformatics Consortium of Taiwan,

NSC100-2319-B-010-002). We thank Ms. Miranda J. Loney for English editing.

\section{Author details}

'Agricultural Biotechnology Research Center, Academia Sinica, 128, Sec. 2, Academia Road, Nankang, Taipei 11501, Taiwan. Institute of Statistical Science, Academia Sinica, Taipei, Taiwan. ${ }^{3}$ Institute of Chemistry, College of Science, University of the Philippines, Quezon City, Philippines. ${ }^{4}$ Miaoli District Agricultural Research and Extension Station, Council of Agriculture, Miaoli, Taiwan. ${ }^{5}$ Institut de Génétique Humaine (CNRS UPR-1142), Montpellier, France. ${ }^{6}$ Department of Life Sciences, National Chung Hsing University, Taichung 402, Taiwan. ${ }^{7}$ Institute of Pharmacology, Yang-Ming University, Taipei 112, Taiwan. ${ }^{8}$ Institute of Zoology, National Taiwan University, Taipei 106, Taiwan.

Received: 10 November 2012 Accepted: 12 February 2013 Published: 23 February 2013

\section{References}

1. Iqbal S, Younas U, Sirajuddin, Chan KW, Sarfraz RA, Uddin K: Proximate composition and antioxidant potential of leaves from three varieties of Mulberry (Morus sp.): a comparative study. Int J Mol Sci 2012, 13(6):6651-6664.

2. Singab AN, El-Beshbishy HA, Yonekawa M, Nomura T, Fukai T: Hypoglycemic effect of Egyptian Morus alba root bark extract: effect on diabetes and lipid peroxidation of streptozotocin-induced diabetic rats. J Ethnopharmacol 2005, 100(3):333-338.

3. Dimo T, Rakotonirina S, Kamgang R, Tan PV, Kamanyi A, Bopelet M: Effects of leaf aqueous extract of Bidens pilosa (Asteraceae) on $\mathrm{KCl}$ - and norepinephrine-induced contractions of rat aorta. J Ethnopharmacol 1998, 60(2):179-182.

4. Doi K, Kojima T, Makino M, Kimura Y, Fujimoto Y: Studies on the constituents of the leaves of Morus alba L. Chem Pharm Bull(Tokyo) 2001, 49(2):151-153

5. Fukai T, Hano Y, Hirakura K, Nomura T, Uzawa J, Fukushima K: Structures of two natural hypotensive diels-alder type adducts, mulberrofuran $\mathrm{F}$ and G, from the cultivated mulberry tree (Morus Ihou Koidz). Chem Pharm Bull (Tokyo) 1985, 33:3195-3204.

6. Lemus I, Garcia R, Delvillar E, Knop G: Hypoglycaemic activity of four plants used in Chilean popular medicine. Phytother Res 1999, 13(2):91-94.

7. Katsube T, Yamasaki M, Shiwaku K, Ishijima T, Matsumoto I, Abe K, Yamasaki $Y$ : Effect of flavonol glycoside in mulberry (Morus alba L.) leaf on glucose metabolism and oxidative stress in liver in diet-induced obese mice. J Sci Food Agric 2010, 90(14):2386-2392.

8. Uno T, Isogai A, Suzuki A, Shirata A: Isolation and Identification of Ethyl B-Resorcylate(Ethyl2,4-Dihydroxybenzoate) and 5,7-Dihydroxychromone from the Root Bark of Mulberry Tree (Morus alba L.) and their Biological Activity. J Sericult Sci Japan 1981, 50(5):422-427. 
9. Park KM, You JS, Lee HY, Baek NI, Hwang JK: Kuwanon G: an antibacterial agent from the root bark of Morus alba against oral pathogens. J Ethnopharmacol 2003, 84(2-3):181-185.

10. Takasugi M, Ishikawa S, Nagao S, Masamune T: Albafuran C, a natural diels-alder adduct of a dehydroprenyl-2-phenylbenzofuran with a chalcone from mulberry. Chem Lett 1982, 8:1223-1224.

11. Rollinger JM, Spitaler R, Menz M, Marschall K, Zelger R, Ellmerer EP, Schneider P, Stuppner H: Venturia inaequalis-inhibiting Diels-Alder adducts from Morus root bark. J Agric Food Chem 2006, 54(22):8432-8436.

12. Du J, He ZD, Jiang RW, Ye WC, Xu HX, But PP: Antiviral flavonoids from the root bark of Morus alba L. Phytochemistry 2003, 62(8):1235-1238.

13. Chai OH, Lee MS, Han EH, Kim HT, Song CH: Inhibitory effects of Morus alba on compound 48/80-induced anaphylactic reactions and antichicken gamma globulin lgE- mediated mast cell activation. Biol Pharm Bull 2005, 28(10):1852-1858.

14. Choi EM, Hwang JK: Effects of Morus alba leaf extract on the production of nitric oxide, prostaglandin E2 and cytokines in RAW264.7 macrophages. Fitoterapia 2005, 76(7-8):608-613

15. Kimura Y, Okuda H, Nomura T, Fukai T, Arichi S: Effects of flavonoids and related compounds from mulberry tree on arachidonate metabolism in rat platelet homogenates. Chem Pharm Bull(Tokyo) 1986, 34(3):1223-1227.

16. Kalantari H, Aghel N, Bayati M: Hepatoprotective effect of Morus Alba L. in carbon tetrachloride-induced hepatotoxicity in Mice. Saudi Pharmaceut $J$ 2009, 17(1):90-94.

17. Oh H, Ko EK, Jun JY, Oh MH, Park SU, Kang KH, Lee HS, Kim YC: Hepatoprotective and free radical scavenging activities of prenylflavonoids, coumarin, and stilbene from Morus alba. Planta Med 2002, 68(10):932-934.

18. Kang TH, Oh HR, Jung SM, Ryu JH, Park MW, Park YK, Kim SY: Enhancement of neuroprotection of mulberry leaves (Morus alba L.) prepared by the anaerobic treatment against ischemic damage. Biol Pharm Bull 2006, 29(2):270-274

19. Bharani SE, Asad M, Dhamanigi SS, Chandrakala GK: Immunomodulatory activity of methanolic extract of Morus alba Linn. (mulberry) leaves. Pak J Pharm Sci 2010, 23(1):63-68.

20. Chandrashekara KT, Nagaraju S, Nandini SU, Kemparaju K: Neutralization of local and systemic toxicity of Daboia russelii venom by Morus alba plant leaf extract. Phytother Res 2009, 23(8):1082-1087.

21. Ali A, Ali M: New triterpenoids from Morus alba L. stem bark. Nat Prod Res 2012. 10.1080/14786419.2012.676547.

22. Kim GN, Jang HD: Flavonol content in the water extract of the mulberry (Morus alba L.) leaf and their antioxidant capacities. J Food Sci 2011, 76(6):C869-C873.

23. Nomura T, Fukai $T$ : Kuwanon $G$, a new flavone derivative from the root barks of the cultivated mulberry tree (Morus alba L.). Chem Pharm Bull (Tokyo) 1980, 28(8):2548-2552.

24. Nomura T, Fukai T, Hano Y: Constituents of the Chinese crude drug Sang-Bai-Pi (Morus Root Bark) I.[1] structure of a new flavanone derivative, Sanggenon A.[2]. Planta Med 1983, 47(1):30-34.

25. Nomura T, Fukai T, Hano Y, Urano S: Constituents of the Chinese Crude Drug Sang-Bai-Pi (Morus Root Bark). Planta Med 1983, 47(2):95-99.

26. Nomura T, Fukai T, Hano Y, Uzawa J: Structure of Sanggenon C, a natural hypotensive diels-alder adduct from Chinese Crude Drug "Sang-bái-pí" (Morus Root Bark). Heterocycles 1981, 16(12):2141-2148.

27. Nomura T, Fukai T, Hano Y, Uzawa J: Structure of Sanggenon D, a Natural Hypotensive Diels-Alder Adduct from Chinese Crude Drug "Sang-bái-pí" (Morus Root Bark). Heterocycles 1982, 17(1):381-389.

28. Nomura T, Fukai T, Katayanagi M: Kuwanon A, B, C and Oxydihydromorusin, four new flavones from the root bark of the cultivated mulberry tree (Morus alba L.). Chem Pharm Bull(Tokyo) 1977, 25(3):529-532.

29. Nomura T, Fukai T, Matsumoto J, Imashimizu A, Terada S, Hama M: Constituents of the cultivated mulberry tree. Planta Med 1982, 46(11):167-174.

30. Nomura T, Fukai T, Narita T: Hypotensive constituent, Kuwanon H, a new flavone derivative from the root bark of the cultivated mylberry tree (Morus alba L.). Heterocycles 1980, 14(12):1943-1951.

31. Nomura T, Fukai T, Uno J, Arai T: Mulberrofuran A, a new isoprenoid 2-Arylbenzofuran from the root bark of the cultivated mulberry tree (Morus alba L.). Heterocycles 1978, 9(11):1593-1601.

32. Nomura T, Fukai T, Yamada S, Katayanagai M: Phenolic constituents of the cultivated mulberry tree (Morus alba L.). Chem Pharm Bull(Tokyo) 1976, 24(11):2898-2900
33. Ueda S, Matsumoto J, Nomura T: Four new natural diels-alder type adducts, Mulberrofuran E, Kuwanon Q, R, and V from Callus culture of Morus Alba L. Chem Pharm Bull(Tokyo) 1984, 32(1):350-353.

34. Ueda S, Nomura T, Fukai T, Matsumoto J: Kuwanon J, a new diels-alder adduct and chalcomoracin from callus culture of Morus Alba L. Chem Pharm Bull(Tokyo) 1982, 30(8):3042-3045.

35. Hano Y, Fukai T, Nomura T, Uzawa J, Fukushima K: Structure of Mulberrofuran I, A Novel 2-arylbenzofura derivative from the cultivated mulberry tree (Morus bombycis Koidz.). Chem Pharm Bull(Tokyo) 1984 32(3):1260-1263.

36. Hano $Y$, Itoh M, Koyoma N, Nomura T: Constituents of the Chinese crude drug Sang-Bai-Pi (Morus Root Bark) V. structures of three new flavanones, Sanggenons L, M, and N. Heterocycles 1984, 22(8):1791-1800.

37. Fukai T, Hano Y, Hirakura K, Nomura T, Uzawa J: Structure of Mulberrofuran $\mathrm{H}$, A Novel 2-arylbenzofuran derivative from the cultivated mulberry tree Morus Ihou (Ser.) Koidz. Chem Pharm Bull(Tokyo) 1984, 32(2):808-811.

38. Jin W, Na M, An R, Lee H, Bae K, Kang SS: Antioxidant compounds from twig of Morus alba. Nat Prod Sci 2002, 8(4):129-132.

39. Chung KO, Kim BY, Lee MH, Kim YR, Chung HY, Park JH, Moon JO: In-vitro and in-vivo anti-inflammatory effect of oxyresveratrol from Morus alba $\mathrm{L}$. J Pharm Pharmacol 2003, 55(12):1695-1700.

40. Barreiro O, Martin P, Gonzalez-Amaro R, Sanchez-Madrid F: Molecular cues guiding inflammatory responses. Cardiovasc Res 2010, 86(2):174-182.

41. Proudfoot A: Chemokine receptors: multifaceted therapeutic targets. Nat Rev Immunol 2002, 2:106-115

42. Moser B, Willimann K: Chemokines: role in inflammation and immune surveillance. Ann Rheum Dis 2004, 63(Suppl 2):ii84-ii89.

43. Kucia M, Jankowski K, Reca R, Wysoczynski M, Bandura L, Allendorf DJ, Zhang J, Ratajczak J, Ratajczak MZ: CXCR4-SDF-1 signalling, locomotion, chemotaxis and adhesion. J Mol Histol 2004, 35(3):233-245.

44. Chien SC, Young PH, Hsu YJ, Chen CH, Tien YJ, Shiu SY, Li TH, Yang CW, Marimuthu P, Tsai LF, et al: Anti-diabetic properties of three common Bidens pilosa variants in Taiwan. Phytochemistry 2009, 70(10):1246-1254.

45. Soekamtoa NH, Achmad SA, Ghisalberti EL, Hakim EH, Syah YM: Lunularin and oxyresveratrol: two stilbene derivatives from Morus macroura. Indo J Chem 2005, 5(3):207-210.

46. Djapic N, Djarmati Z, Filip S, Jankov RM: A stilbene from the heartwood of Maclura pomifera. J Serb Chem Soc 2003, 68(3):235-237.

47. Shen MY, Liu YJ, Don MJ, Liu HY, Chen ZW, Mettling C, Corbeau P, Chiang CK, Jang YS, Li TH, et al: Combined phytochemistry and chemotaxis assays for identification and mechanistic analysis of anti-inflammatory phytochemicals in Fallopia japonica. PLoS One 2011, 6(11):e27480.

48. Medina-Holguin AL, Holguin FO, Micheletto S, Goehle S, Simon JA, O'Connell MA: Chemotypic variation of essential oils in the medicinal plant, Anemopsis californica. Phytochemistry 2008, 69(4):919-927.

49. Nyman T, Julkunen-Tiitto $R$ : Chemical variation within and among six northern willow species. Phytochemistry 2005, 66(24):2836-2843.

50. Chen $\mathrm{CH}$ : Generalized association plots for information visualization: the applications of the convergence of iteratively formed correlation matrices. Statistica Sinica 2002, 12:1-23.

51. Tien YJ, Lee YS, Wu HM, Chen CH: Methods for simultaneously identifying coherent local clusters with smooth global patterns in gene expression profiles. BMC Bioinformatics 2008, 9:155.

52. Murdoch $C$, Finn $A$ : Chemokine receptors and their role in inflammation and infectious diseases. Blood 2000, 95(10):3032-3043.

53. Rozengurt E: Mitogenic signaling pathways induced by $\mathrm{G}$ proteincoupled receptors. J Cell Physiol 2007, 213(3):589-602.

54. Yang CL, Chik SC, Li JC, Cheung BK, Lau AS: Identification of the bioactive constituent and its mechanisms of action in mediating the antiinflammatory effects of black cohosh and related Cimicifuga species on human primary blood macrophages. J Med Chem 2009, 52(21):6707-6715.

\section{doi:10.1186/1472-6882-13-45}

Cite this article as: Chen et al:: Morus alba and active compound oxyresveratrol exert anti-inflammatory activity via inhibition of leukocyte migration involving MEK/ERK signaling. BMC Complementary and Alternative Medicine 2013 13:45. 